\title{
YBaCuO ceramics-based DC interferometer with current-formed weak links
}

\author{
S I BONDARENKO, A V LUKASHENKO, A A SHABLO, \\ E N TILCHENKO and Yu K NEIMIROK \\ Institute for Low Temperature Physics and Engineering, UkrSSR Academy of Sciences, \\ Kharkov 310164, USSR
}

\begin{abstract}
The results of the study of dc interferometers and SQUIDS from the thick-film $\mathrm{Y}_{1} \mathrm{Ba}_{2} \mathrm{Cu}_{3} \mathrm{O}_{7-\delta}$ ceramics are presented here. It is shown that under the influence of high-density currents through the film constriction the differential resistance of interferometers thus formed increases by $2-3$ orders of magnitude and the voltage-field characteristics become more regular. The frequency dependence of intrinsic noise in dc SQUIDs based on thick-film interferometers is investigated. It is demonstrated that in the white noise frequency region (1-10 Hz) the energy sensitivity is within $\delta \varepsilon_{n}=10^{-28}$ to $10^{-29} \mathrm{~J} / \mathrm{Hz}$.
\end{abstract}

Keywords. SQUIDs; quantum interference; films; high-temperature ceramics; noise.

To create a dc SQUID with a working temperature of $77 \mathrm{~K}$, a study was made of macroscopic quantum interference in singly-connected samples of thick-film high-temperature superconducting (HTSC) $\mathrm{Y}-\mathrm{Ba}-\mathrm{Cu}-\mathrm{O}$ ceramics.

The current-voltage characteristics (IVC) and voltage-field characteristics (VFC) of samples were measured at d.c. according to the four-probe technique. The current and potential wires were connected to the samples with indium applied by rubbing into the HTSC ceramic surface and glued with silver paste. The noise spectrum in the frequency range 0.01 to $25 \mathrm{~Hz}$ was studied by a Fourier spectrum analyser based on microcomputer DVK-2, and in the range 1 to $1000 \mathrm{~Hz}$ by a nanovoltmeter "Unipan". To decrease the external magnetic field effect, the interferometers under investigation were enclosed in a triple-layer permalloy shield. The external rf fields were reduced by a niobium shield.

Thick-film $\mathrm{Y}_{1} \mathrm{Ba}_{2} \mathrm{Cu}_{3} \mathrm{O}_{7-\delta}$ samples were prepared on $\mathrm{ZrO}_{2}$ substrates by silk screen-printing (Bondarenko et al 1989). The HTSC coating thickness was $50-100 \mu \mathrm{m}, T_{\mathrm{c}} \approx 95 \mathrm{~K}$, the superconducting transition width was 1.5 to $2 \mathrm{~K}$. The critical current density was $I_{c}=4 \times 10^{2}-10^{3} \mathrm{~A} / \mathrm{cm}^{2}$. The sample configuration is shown on the inset to figure 1 . A substrate of $2 \times 5 \mathrm{~mm}^{2}$ was cut by the diamond wheel out of a $\mathrm{ZrO}_{2}$ plate with a thick $\mathrm{Y}_{1} \mathrm{Ba}_{2} \mathrm{Cu}_{3} \mathrm{O}_{7-\delta}$ film applied on the surface. A constriction of $0.3 \times 0.15 \mathrm{~mm}^{2}$ was made in the central part of the film.

The voltage-field characteristics of the samples in the initial state at $77 \mathrm{~K}$ were of intricate shape, so the samples were acted upon by a high-density $\left(5 \times 10^{4} \mathrm{~A} / \mathrm{cm}^{2}\right) \mathrm{AC}$ of $50 \mathrm{~Hz}$ which was passed through the constriction in liquid nitrogen. This causes a decrease of their critical current $I_{c}$ and a 2-3 orders of magnitude increase of their differential resistance $\left(R_{d}\right)$. 


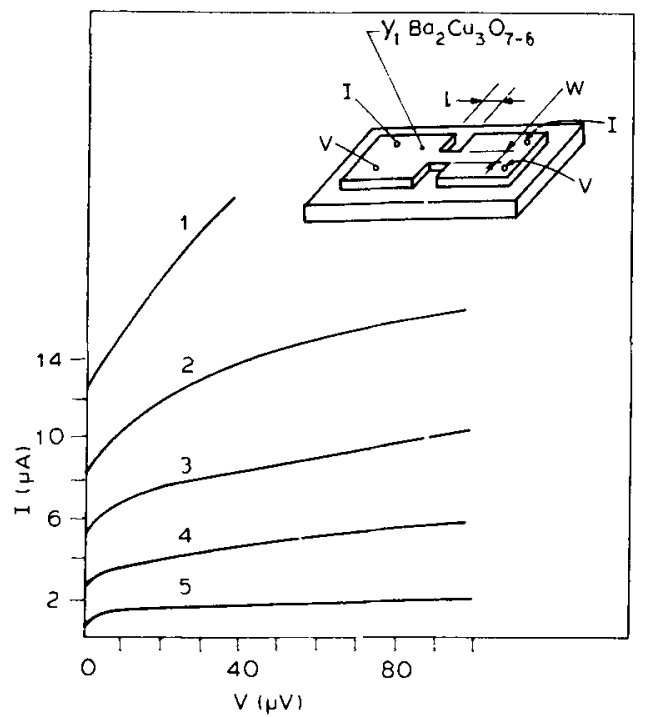

Figure 1. Current-voltage characteristics of thick-film sample No $140 / 1$ at $T=77 \mathrm{~K}$. 1 before the AC action on the sample; 2,3,4,5-IVC taken in the process of sample parameters adjustment by high-density alternating current. Inset: Sample with a constriction (bridge).

Figure 1 shows IVC of sample No $140 / 1$ at $T=77 \mathrm{~K}$ taken in the process of controlling the characteristics by current. One can clearly see the $R_{d}$ increase with the decrease of $I_{c}$. At microwave irradiation with a frequency of $10 \mathrm{GHz}$ the IVC of the samples reveal Josephson current steps. With the decrease in $I_{c}$, the extent of microwave power effect on the samples increases, the IVC current steps become more distinct and grow in number. The a.c. effect results in distinguishing one or two VFC periods corresponding to the quantizing loop areas of $10^{2}$ to $10^{4} \mu \mathrm{m}^{2}$.

Figure 2 shows typical VFC for three $(a, b, c)$ samples acted upon by alternating current.

Figure 3 shows the results of noise characteristics in two DC SQUIDs based on thick-film HTSC interferometers produced according to the above technique. The measurements were made at $77 \mathrm{~K}$ in the flux-locked loop mode. In the region of $f \geqslant 10 \mathrm{~Hz}$ the magnetic flux noise $\Phi_{n}$ of the SQUID is frequency-independent and for sample No $142 / 3$ is $\Phi_{n} \approx 10^{-4} \Phi_{0} / \mathrm{Hz}^{1 / 2}$, for sample No $142 / 5 \Phi_{n} \approx 5 \times 10^{-5} \Phi_{0} / \mathrm{Hz}^{1 / 2}$ which, in the units of energy, corresponds to $\delta \varepsilon_{n}=10^{-28} \mathrm{~J} / \mathrm{Hz}$ and $\delta \varepsilon_{n}=3 \times 10^{-29} \mathrm{~J} / \mathrm{Hz}$ at the interferometer inductance $L_{0} \approx 1.4 \times 10^{-10} \mathrm{H}$ and $1.6 \times 10^{-10} \mathrm{H}$ determined from the relation $\Delta I_{c} L_{0}=\Phi_{0} / 2$, where $\Delta I_{c}$ is the depth of critical current modulation of the interferometer, $\Phi_{0}$ is the magnetic flux quantum.

It is seen that the DC SQUID noise at $77 \mathrm{~K}$ is sufficiently low and it is close to commercial rf SQUID noises with pressure contacts at $4.2 \mathrm{~K}$. Since the $L_{0}$ value of a two-contact interferometer determined from the $\Delta I_{c}$ can substantially depend on the critical current difference of contacts, the inductance was also determined by calculations. Using the formula $L_{0}=\mu_{0} \mathrm{RF} / 2 \pi$ (Kalantarow and Tseitlin 1986), where $\mu_{0}=4 \pi \times 10^{-7} \mathrm{H} / \mathrm{m}, R \approx 50 \mu \mathrm{m}$ is the effective radius of the quantizing loop and $\mathrm{F}$ the coefficient determined from the table, we obtain for sample No $142 / 5 L_{0} \approx 1.2 \times 10^{-10} \mathrm{H}$ 


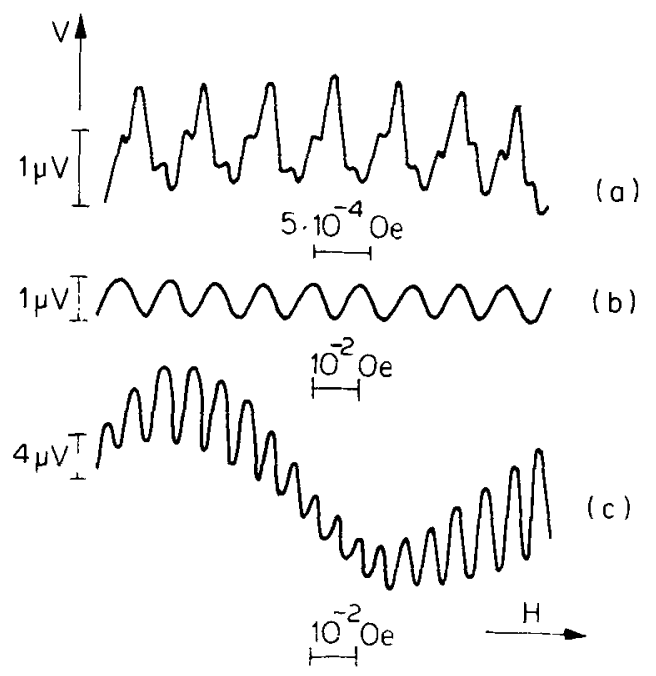

Figure 2. Voltage-field characteristics of thick-film DC interferometers after the action of high density alternating current. a. No. $142 / 9, I_{c}(77 \mathrm{~K})=47 \mu \mathrm{A} ;$ b. No $140 / 2, I_{c}(77 \mathrm{~K})=$ $13 \mu \mathrm{A}$; c. No $142 / 3, I_{c}(77 \mathrm{~K})=10 \mu \mathrm{A}$.

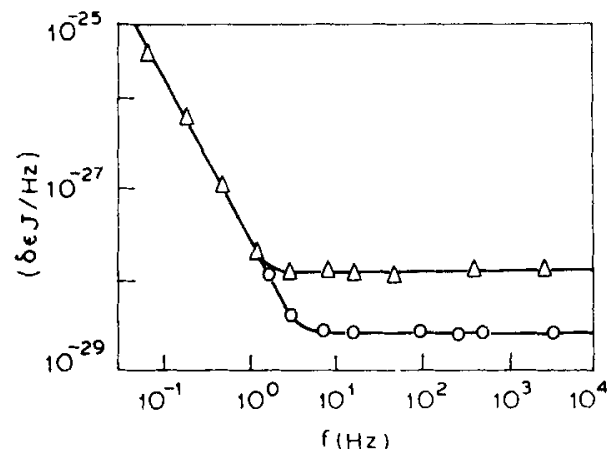

Figure 3. Frequency dependence of the spectral density of noise in SQUIDs with thick-film interferometers No $142 / 3$ and No $142 / 5, T=77 \mathrm{~K}$.

It is logical to assume that the quantizing loop shape is close to symmetrical one as its size $2 R \approx \sqrt{S}$ practically coincides with the transverse dimension of the sample constriction $(120 \mu \mathrm{m})$ and $S$ is the constriction area in plan. However, even if the quantizing loop is elongated, and its length $l$ ratio to width $W$ (at the same area $S$ ) $l / W=4$, it results in the inductance determination error not greater than $17 \%$.

Two features deserve attention. First, in contract to our data, the noise in the bulky DC interferometer (Lam Chock Sing et al 1989) increases with decrease of frequency over the whole frequency range under investigation. Secondly, in the low-frequency range $(f \leqslant 1 \mathrm{~Hz})$ a thick-film interferometer has a lower intrinsic noise than a bulky one. Thus, at $f \approx 1 \mathrm{~Hz}$ the energy resolution of various samples from bulky HTCP ceramics lies within $5 \cdot 10^{-27}-2 \cdot 10^{-26} \mathrm{~J} / \mathrm{Hz}$, whereas the thick-film samples at this 
frequency reveal $4 \cdot 10^{-28} \mathrm{~J} / \mathrm{Hz}$, i.e. 10 to 50 times better. The origin of these differences should be probably sought for in the $1 / f$ noise sources in HTSC interferometers. One of the most probable causes of $1 / f$ noise is the motion of magnetic flux trapped by the interferometer which depends on the structural characteristics of the interferometer material and, therefore, will be different in bulky samples and thin films. The fine-crystalline thick films with a large number of pores and intergranular boundaries have probably a greater pinning than denser bulky samples, hence the noise in thick films should be lower.

The SQUID noise is determined by two factors: The noise of electronic equipment $\left(\delta \Phi_{n}^{e}\right)$ and the noise of interferometer proper $\left(\delta \Phi_{n}^{i}\right)$ characterized by the normal $(R)$ and dynamic $\left(R_{d}\right)$ resistances.

The measured voltage fluctuations of amplifiers and converters do not exceed $V_{n}^{e}=3 \cdot 10^{-11} \mathrm{~V} / \mathrm{Hz}^{1 / 2}$.

The interferometer noise voltage was estimated by the relation (Vystavkin et al 1974)

$$
V_{n}^{i}=\left[1+\frac{1}{2}\left(\frac{I_{c}}{I_{0}}\right)^{2}\right] \frac{4 k R_{d}^{2} T}{R}
$$

where $I_{c}$ is the critical current and $I_{0}$ is the interferometer operating current (typically $\left.I_{0} / I_{\mathrm{c}} \approx 1 \cdot 3\right)$. For sample No $142 / 3 V_{n}^{i} \approx 1 \cdot 25 \times 10^{-10} \mathrm{~V} / \mathrm{Hz}^{1 / 2}$. Using $\delta \Phi_{n} \approx V_{n} /(\delta V / \delta \Phi)$; $V_{n}=V_{n}^{i}+V_{n}^{e}, \delta V / \delta \Phi \approx V_{0} / \Phi_{0}$, the steepness of flux conversion into voltage; $V_{0}$, amplitude of oscillations $V(H)$, we will obtain at $V_{0}=5 \cdot 10^{-6} \mathrm{~V} \delta \Phi \approx 5 \cdot 10^{-6} \Phi_{0} / \mathrm{Hz}^{1 / 2}$ which is less than the value characterizing the plateau on figure 3 (for the given sample $\delta \Phi_{n} \approx 10^{-4} \Phi_{0} / \mathrm{Hz}^{1 / 2}$ ). A similar situation is observed for sample No $142 / 5$. This suggest the presence of an additional source of noise in the thick-film HTSC interferometers.

Lam Chock Sing et al (1989) pointed to the instability in time of the characteristics of already adjusted samples of bulky $\mathrm{Y}-\mathrm{Ba}-\mathrm{Cu}-\mathrm{O}$ ceramics. This is confirmed by our studies. On the other hand some of the thick-film samples practically did not change their characteristics within nine months, which can be attributed to both the stabilizing effect of the substrate and the distinctions in the $\mathrm{Y}-\mathrm{Ba}-\mathrm{Cu}-\mathrm{O}$ ceramics manufacture.

In conclusion, a bridge-type construction in a thick $\mathrm{Y}_{1} \mathrm{Ba}_{2} \mathrm{Cu}_{3} \mathrm{O}_{7-\delta}$ film affected with a high-density control current in liquid nitrogen displays all the properties of a DC interferometer. In this case the repeated actions of the control current cause the differential resistance increase of the constriction, critical current decrease at $T=77 \mathrm{~K}$ and simplification of quantum oscillations of the interferometer voltage dependence on a magnetic field normal to the film surface. The intrinsic noise of the SQUID based on these interferometers is sufficiently low at $T=77 \mathrm{~K}$ and is close to the noise of commercial RF SQUIDs at $T=4 \cdot 2 \mathrm{~K}$.

The authors express their gratitude to $\mathrm{GE}$ Vedernikov for providing thick-film HTSC coatings.

\section{References}

Bondarenko S I et al 1989 Sverkhprovodimost 297

Kalantarov P L and Tseitlin L O 1986 in Calculation of inductances (Leningrad: Energoatomizdat) p. 448 Lam Chock Sing M et al 1989 IEEE Trans. Mag. 25889

Vystavkin A N et al 1974 Rev. Phys. Appl. 979 